\title{
Prevalence and Predictors for Homo- and Heterosubtypic Antibodies Against Influenza A Virus
}

\author{
Ines Kohler, ${ }^{1,2}$ Alexandra U. Scherrer, ${ }^{3, a}$ Osvaldo Zagordi, ${ }^{1, a}$ Matteo Bianchi, ${ }^{1}$ Arkadiusz Wyrzucki, ${ }^{1,2}$ Marco Steck, ${ }^{1}$ \\ Bruno Ledergerber, ${ }^{3}$ Huldrych F. Günthard, ${ }^{3}$ and Lars Hangartner ${ }^{1}$ \\ ${ }^{1}$ Institute of Medical Virology, University of Zurich, ${ }^{2}$ Microbiology and Immunology, Life Science Zurich Graduate School, and ${ }^{3}$ Division of Infectious \\ Diseases and Hospital Epidemiology, University Hospital Zurich, University of Zurich, Switzerland
}

Background. The effectiveness of trivalent influenza vaccination has been confirmed in several studies. To date, it is not known whether repeated exposure and vaccination to influenza promote production of cross-reactive antibodies. Furthermore, how strains encountered earlier in life imprint the immune response is currently poorly understood.

Methods. To determine the prevalence for human homo- and heterosubtypic antibody responses, we scrutinized serum samples from 305 healthy volunteers for hemagglutinin-binding and -neutralizing antibodies against several strains and subtypes of influenza A. Statistical analyses were then performed to establish the association of measured values with potential predictors.

Results. It was found that vaccination not only promoted higher binding and neutralizing antibody titers to homosubtypic influenza isolates but also increased heterosubtypic human immune responses. Both binding and neutralizing antibody titers in relation with age of the donors mirrored the course of the different influenza strain circulation during the last century. Advanced age appeared to be of advantage for both binding and neutralizing titers to most subtypes. In contrast, the first virus subtype encountered was found to imprint to some degree subsequent antibody responses. Antibodies to recent strains, however, primarily seemed to be promoted by vaccination.

Conclusions. We provide evidence that vaccinations stimulate both homo- and heterosubtypic immune responses in young and middle-aged as well as more senior individuals. Our analyses suggest that influenza vaccinations not only prevent infection against currently circulating strains but can also stimulate broader humoral immune responses that potentially attenuate infections with zoonotic or antigenically shifted strains.

Keywords. influenza virus; heterosubtypic antibodies; prevalence; predictors.

The antibody response to influenza viruses is highly strain- and subtype-specific, and is primarily directed against the variable immunodominant apical epitopes on the hemagglutinin (HA) protein [1]. Vaccine-

Received 9 May 2014; accepted 12 August 2014; electronically published 18 August 2014.

${ }^{\mathrm{a}}$ A. U. S. and O. Z. contributed equally to this work.

Correspondence: Lars Hangartner, PhD, Institute of Medical Virology, University of Zurich, Winterthurerstrasse 190, Zurich, Switzerland (hangartner.lars@virology. uzh.ch).

Clinical Infectious Diseases ${ }^{\circledR} \quad$ 2014;59(10):1386-93

(C) The Author 2014. Published by Oxford University Press on behalf of the Infectious Diseases Society of America. All rights reserved. For Permissions, please e-mail: journals.permissions@oup.com.

DOl: $10.1093 /$ cid/ciu660 induced antibodies are therefore only effective against viruses closely related to the inoculated strains, and immunization needs to be revised annually to reflect the antigenic outfit of the viruses predicted to be predominant in the following influenza season. The breadth of the anti-influenza virus antibody response is further limited by the original antigenic sin in that every immunization also boosts the memory response to the priming strain [2]. Indeed, Lessler and colleagues stated that in humans, repeated exposure to different $\mathrm{H} 3 \mathrm{~N} 2$ strains increased antibody titers to strains encountered earlier in life [3].

Heterosubtypic antibodies-that is, antibodies recognizing HAs from different subtypes-are rare [4]: In human prevaccination sera, only $0.01 \%$ of total serum 
Table 1. Cohort Characteristics $(\mathbf{N}=\mathbf{3 0 5})$

\begin{tabular}{lc}
\hline Characteristic & No. (\%) or Median [IQR] \\
\hline Sex, female & $156(51.15)$ \\
Age, y & $36[28-49]$ \\
No. of vaccinations & $2[0-5]$ \\
No. of episodes & $2[0-3]$ \\
Contact to FPV-infected poultry & $3(0.98)$ \\
Travel to rural region in southeastern Asia & $23(7.54)$ \\
Novartis vaccine trial & 17 (excluded) \\
"Swine flu" (H1pdm/09) infection & 0 \\
\hline
\end{tabular}

Abbreviations: FPV, fowl plague virus; IQR, interquartile range.

immunoglobulin $\mathrm{G}$ has been described to have heterosubtypic binding activity, one-tenth of which is specific for the HA stem epitope [4-6]. To establish the prevalence and predictors for heterosubtypic antibody responses, serum from 305 human immunodeficiency virus-negative volunteers was collected in October 2009. In these serum samples, we assessed both binding to 5 human and 3 nonhuman influenza isolates, and neutralizing antibody titers to 5 human and 4 nonhuman influenza isolates. These data were put into relation with epidemiological information acquired with a questionnaire at the time of the blood draw.

\section{METHODS}

\section{Procedures}

Sera from 305 randomly selected healthy volunteers were prospectively collected in Zurich, Switzerland, in late 2009 before the arrival of the influenza A(H1N1) 2009 pandemic strain (H1pdm/ 09). The study was approved by the ethics committee of the University Hospital Zurich and written informed consent was obtained from all study participants. Self-reported demographic data including age, sex, travel history, the total number of estimated previous influenza vaccinations, estimated times of influenza infections (defined as "flu" with $>3$ bedridden days and high fever), and potential contact to influenza-infected birds were collected on the day of the blood draw (an English translation of the questionnaire is given in the Supplementary Methods). As antibodies binding to avian or extinct human HA subtypes are bona fide cross-reactive, we determined binding antibody titers to recombinant trimeric HA protein from $\mathrm{rH} 1 \mathrm{pdm} / 09, \mathrm{rH} 2 / 57$,

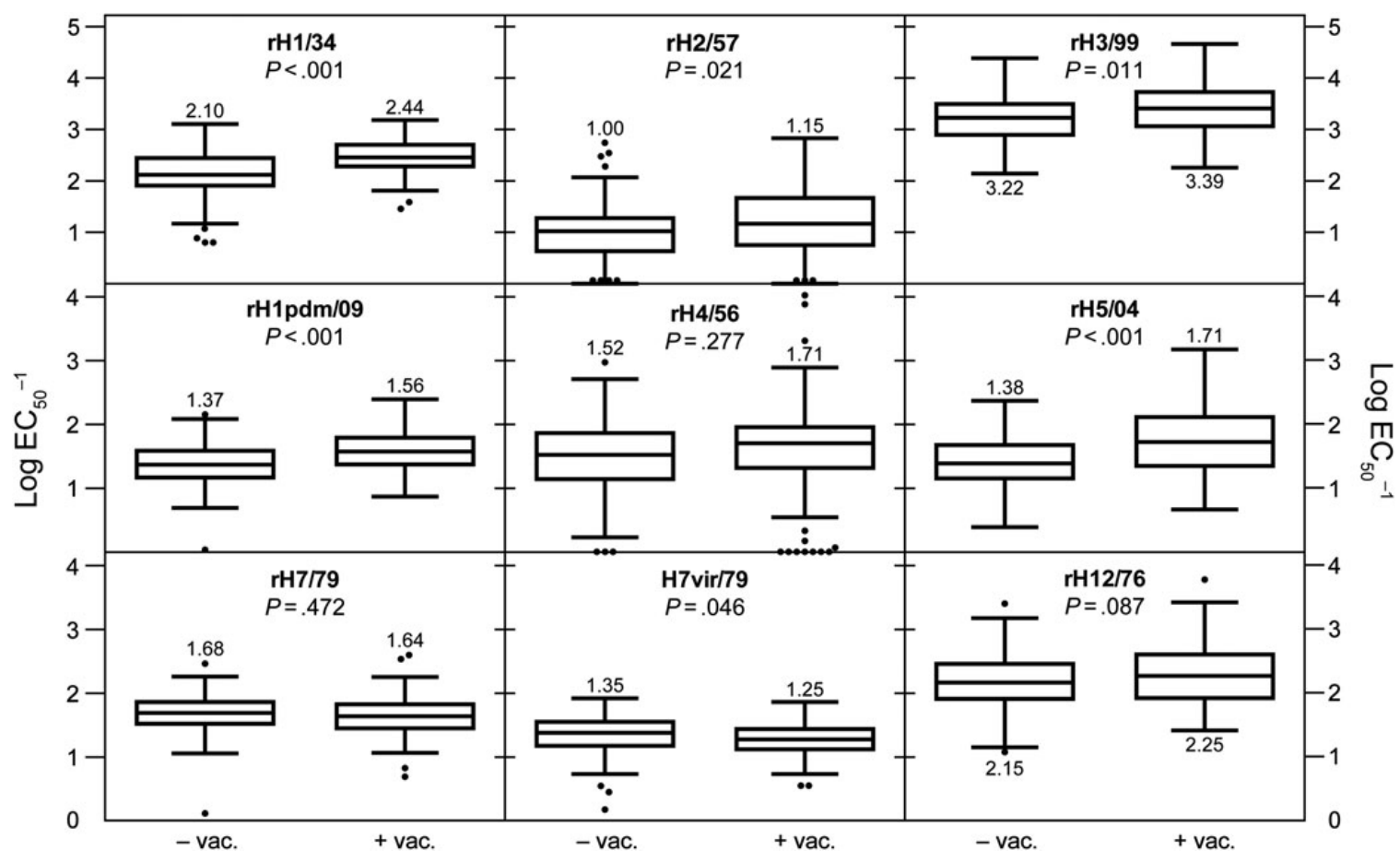

Figure 1. Vaccination-dependent differences in binding antibody titers $\left(-\log \left[\mathrm{EC}_{50}\right]\right)$ against recombinant human and avian influenza hemagglutinin. Serum antibody reactivities to the indicated immobilized recombinant hemagglutinins were assessed by enzyme-linked immunosorbent assay. Two-sample $t$ test analyses of the data were performed and showed higher binding antibody titers to the human subtypes $\mathrm{rH} 1 / 34$ and rH3/99, to heterosubtypic rH1pdm/09 and $\mathrm{rH} 2 / 57$, and to avian heterosubtypic $\mathrm{rH} / 04$ titers in the vaccinated cohort. Values are shown as logarithm of the reciprocal serum dilution giving a halfmaximal signal $\left(-\log \left[\mathrm{EC}_{50}\right]\right)$. Median and $P$ values of each group are indicated by numbers. Box plots represent median and interquartile range (IOR); whiskers depict lower or upper quartile $\pm 1.5 \times \mathrm{IQR}$. Abbreviation: $\mathrm{EC}_{50}$, half-maximal effective concentration. 
$\mathrm{rH} 4 / 56, \mathrm{rH} 5 / 04, \mathrm{rH} 7 / 79$, and $\mathrm{rH1} / 76$ by enzyme-linked immunosorbent assay (ELISA) and put them into relation to homotypic antibodies to $\mathrm{rH} 1 / 34$ and $\mathrm{rH} 3 / 99$. In addition, we assessed whether antibodies binding to recombinant protein can also bind immobilized purified H7N7 virions (H7vir/79; Supplementary Figure 3). The half-maximal effective dose $\left(\mathrm{EC}_{50}\right)$ was then determined by nonlinear regression of optical density values and used for statistical analyses as well as to establish correlations with the information collected in the questionnaire.

Strains used for neutralization assays are described in the Supplementary Data. For evaluation, the reciprocal 50\% inhibitory dose was determined by nonlinear regression to the logarithm of serum concentration $\left(-\log \left[\mathrm{IC}_{50}\right]\right)$, where possible. If most samples did not reach signal saturation, and constrained nonlinear regression would have been prone for fitting artifacts, the inhibition percentage at the second serum dilution (1:90) was taken as a surrogate value. Statistical analyses were performed as outlined in the Supplementary Methods. R scripts used for this study are available at https://github.com/ozagordi/FluAbs.

\section{RESULTS}

For the analysis of the prevalence and predictors of heterosubtypic antibodies, a random study population was compiled $(n=305$; Table 1). At a serum dilution corresponding to the detection level of natural antibodies [7] and a relaxed threshold (3-fold over background), between $99 \%$ and $100 \%$ of individuals, depending on the antigen tested, scored positive for serum antibody binding, including all heterosubtypic HAs. Also at more stringent conditions of 1 in 90, 98\%-100\% of individuals scored positive. If a more stringent threshold of a half-maximal signal was applied, heterosubtypic specificity could be monitored in 32\%-99\% (at 1 in 30) and $0.3 \%-79 \%$ (at 1 in 90 ) of the participants. The corresponding values are depicted in Supplementary Figure 1.

With the exception of $\mathrm{rH} 12 / 76$, binding titers to the avian strains ( $\mathrm{rH} 4 / 56, \mathrm{rH} 5 / 04$, and $\mathrm{rH} 7 / 79)$ were low compared with human HAs. Low titers were also observed against $\mathrm{rH} 1 \mathrm{pdm} / 09$ that had not yet arrived in Switzerland when the blood was collected. Titers against human $\mathrm{rH} 2 / 57$ that became

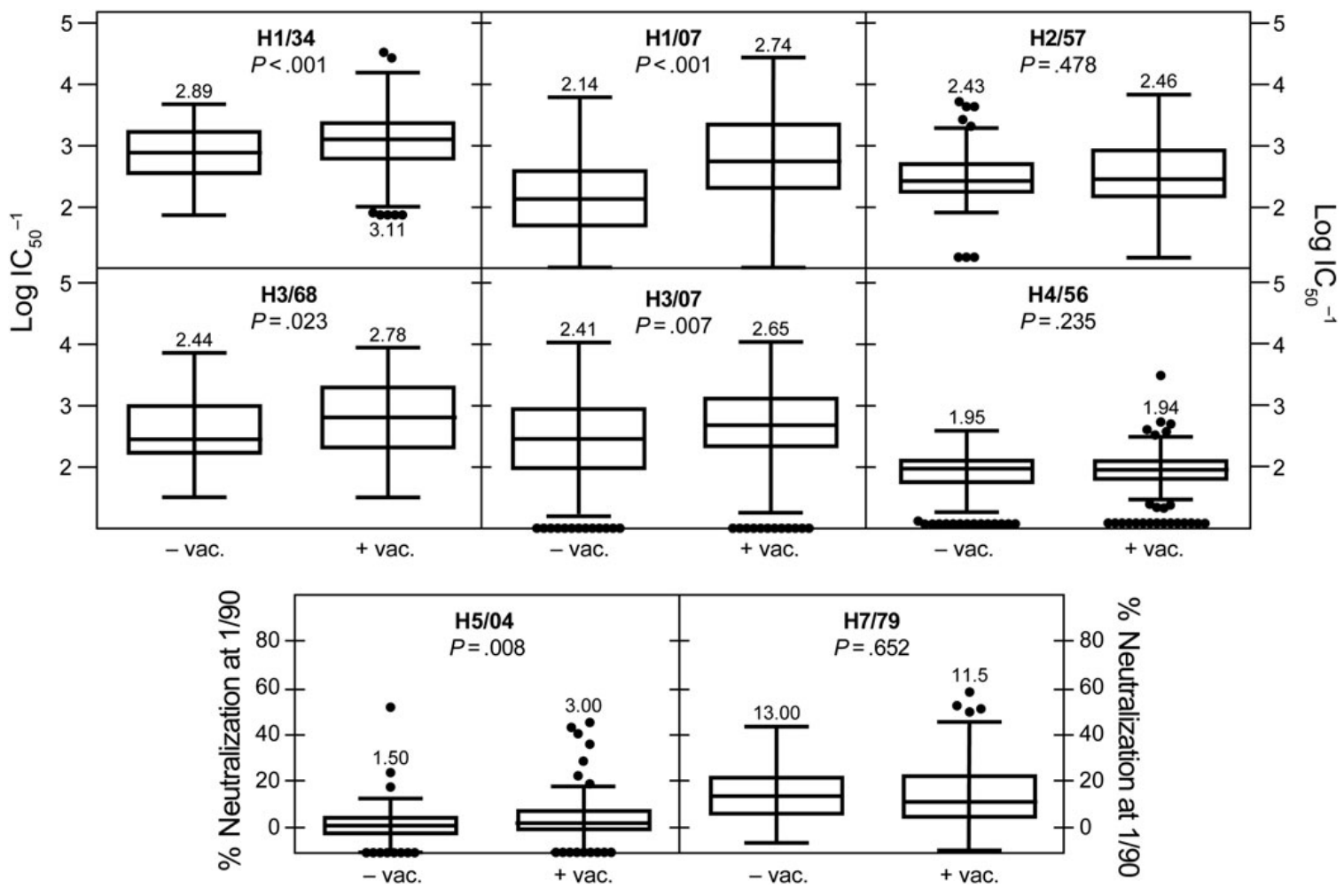

Figure 2. Differences in serum half-maximal inhibitory titer $-\log \left(I_{50}\right)$ in vaccinated and nonvaccinated individuals against human and avian influenza subtypes. Serum antibody neutralizations against the indicated viral subtypes were assessed in neutralization assays. Two sample $t$ test analysis were performed and showed higher neutralizing antibody titers to all homotypic viruses ( $\mathrm{H} 1 / 07, \mathrm{H1} / 34, \mathrm{H3} / 68$, and $\mathrm{H3} / 07$ ) and to heterosubtypic avian virus $\mathrm{H} 5$ / $\mathrm{O} 4$ in the vaccinated cohort. No improvement in titers for human heterosubtypic $\mathrm{H} 2 / 57$ and for avian $\mathrm{H} 4 / 56$ or $\mathrm{H} 7 / 79$ viruses was found. Values are shown as logarithm of the reciprocal serum dilution giving a half-maximal inhibitory concentration $\left(\mathrm{IC}_{50}\right)$, and as inhibition percentage against $\mathrm{H5} / 57$ and $\mathrm{H} 7 / 79$ at a serum dilution of 1 in 90 . Median and $P$ values are indicated. Box plots indicate median and interquartile range (IQR); whiskers include lower or upper quartile $\pm 1.5 \times 10 R$. 
Table 2. Linear Regression Analyzing the Impact of Age and Vaccination on Binding and Neutralizing Antibody Titers

\begin{tabular}{|c|c|c|c|c|c|}
\hline Subtype & rHAs and Strains & Intercept & Age $(\alpha)$ & Vaccination $(\beta)$ & Interaction $(\gamma)$ \\
\hline \multicolumn{6}{|l|}{ Human } \\
\hline \multirow[t]{3}{*}{ Binding } & $\mathrm{rH} 1 / 34$ & $1.80 \pm 0.067$ & $0.0081 \pm 0.0017$ & $0.33 \pm 0.041$ & \\
\hline & $\mathrm{rH} 2 / 57$ & & $0.029 \pm 0.0007$ & & \\
\hline & $\mathrm{rH} 3 / 99$ & $3.58 \pm 0.099$ & $-0.0096 \pm 0.0025$ & $0.21 \pm 0.062$ & \\
\hline \multirow[t]{5}{*}{ Neutralization } & $\mathrm{H} 1 / 34$ & $2.92 \pm 0.043$ & & $0.21 \pm 0.054$ & \\
\hline & $\mathrm{H} 1 / 07$ & $2.34 \pm 0.070$ & & $0.54 \pm 0.087$ & \\
\hline & $\mathrm{H} 2 / 57$ & $1.83 \pm 0.12$ & $0.019 \pm 0.0032$ & $-0.55 \pm 0.16$ & $0.012 \pm 0.0040$ \\
\hline & $\mathrm{H} 3 / 68$ & $1.64 \pm 0.097$ & $0.027 \pm 0.0024$ & & \\
\hline & $\mathrm{H} 3 / 07$ & $2.47 \pm 0.065$ & & $0.24 \pm 0.082$ & \\
\hline \multicolumn{6}{|l|}{ Heterosubtypes } \\
\hline \multirow[t]{5}{*}{ Binding } & $\mathrm{rH} 1 \mathrm{pdm} / 09$ & $1.19 \pm 0.065$ & $0.0047 \pm 0.0016$ & $0.18 \pm 0.041$ & \\
\hline & $\mathrm{rH} 4 / 56$ & $1.62(\mathrm{IQR}=0.68)$ & & & \\
\hline & $\mathrm{rH} 5 / 04$ & $0.93 \pm 0.099$ & $0.013 \pm 0.0025$ & $0.27 \pm 0.062$ & \\
\hline & $\mathrm{rH} 7 / 79$ & $1.64(\mathrm{IQR}=0.36)$ & & & \\
\hline & $\mathrm{rH} 12 / 76$ & $2.22(\mathrm{IQR}=0.62)$ & & & \\
\hline \multirow[t]{2}{*}{ Neutralization } & $\mathrm{H} 5 / 04$ & $1.36 \pm 1.05$ & & $3.62 \pm 1.34$ & \\
\hline & $\mathrm{H} 7 / 79$ & $11.5(\mathrm{IOR}=17.5)$ & & & \\
\hline
\end{tabular}

Binding titers $-\log \left(\mathrm{EC}_{50}\right)$ and neutralizing titers $-\log \left(\mathrm{IC}_{50}\right)$ were modeled as a linear function of age, vaccination, and their interaction (intercept $+\alpha \times$ age $+\beta \times$ vaccination $+\gamma \times$ age $\times$ vaccination). The age is given in years and vaccination is a binary dummy variable. Starting from the complete model, coefficients not significantly different from zero (at the $1 \%$ level) were dropped and the model estimated again. The table reports the parameter estimates for the final model. Where no effect of age and/or vaccination was detected, median and interquartile range are reported in the intercept column.

Abbreviations: $\mathrm{EC}_{50}$, half-maximal effective concentration; $\mathrm{IC}_{50}$, half-maximal inhibitory concentration; IQR, interquartile range; rHAs, recombinant hemagglutinins.

extinct in 1968 were also found to be relatively low, although distributed over a wide range of values.

To determine the direct antiviral activity of these antibodies, we performed in vitro neutralization assays against $\mathrm{H} 1 / 34, \mathrm{H} 1$ / 07, $\mathrm{H} 2 / 57, \mathrm{H} 3 / 07, \mathrm{H} 3 / 68, \mathrm{H} 4 / 56, \mathrm{H} 5 / 04$, and $\mathrm{H} 7 / 79$ viruses. The highest neutralizing titers were found against homologous human strains (H1/34, H1/07, H2/57, H3/68, and H3/07; Supplementary Figure $1 C$ and Supplementary Table 1). Titers to avian H4/56 were much lower, and the barely detectable neutralizing activity to $\mathrm{H} 5$ and $\mathrm{H} 7$ even prevented computation of the $\log \left(\mathrm{IC}_{50}\right)$ values. Instead, the percentage of inhibition at the first dilution ( 1 in 90) was taken as a comparative proxy value for these viruses (Supplementary Figure $1 D$ ).

ELISA titers and neutralizing activity correlated well in that high titers in ELISA are indicative for high neutralizing activity (Supplementary Table 2). Vaccinated individuals clearly displayed higher binding and neutralizing titers against human subtypes $\mathrm{H} 1 / 34$, while still showing increased titers against $\mathrm{rH} 2 / 57, \mathrm{rH} 3 / 99$, and H3/68 (Figures 1 and 2), albeit less markedly. As highlighted in the regression analysis, some of these differences may be explained by age alone (Table 2). Vaccinated individuals also displayed significantly higher neutralizing titers against $\mathrm{H} 1 / 07$ and $\mathrm{H} 3 / 07$ than nonvaccinated individuals. They also displayed higher binding antibody titers to heterosubtypic rH1pdm/09 and rH5/04 (Figure 1). Vaccination showed no or weaker effect on binding titers against the remaining 3 tested heterosubtypic rHAs.

The effect of age, influenza episodes, and sex was also analyzed (Tables 3 and 4). As an exploratory data analysis, we stratified the age in 3 different categories according to the different influenza pandemics: baseline, born 1969-2009 (age 0-40); category 1, born 1958-1968 (age 41-51); category 2, born 19191957 (age 52-90). After adjusting the model for age, influenza episodes, and sex, vaccination still remained a predictor for higher binding and neutralizing titers to the same subtypes as in the two-sample $t$ test. However, the impact of vaccination was lost for $\mathrm{rH} 2 / 57$ binding and $\mathrm{H} 3 / 68$ neutralizing titers in the adjusted models (Tables 3 and 4).

We then performed linear regression to correlate the age of donors and the vaccination status with binding and neutralizing antibody titers (Supplementary Figure 2 and Table 2).

Binding titers to human strains all increased with age, while increasing only in 2 of 5 nonhuman strains, all of these phylogenetically close to the human ones (H1pdm/09 and H5/04). Neutralizing titers were also found to increase with age in 2 of 5 human strains tested (H2/57 and H3/68). In contrast, titers to heterosubtypic strains did not display a dependence on age.

Similarly, vaccination showed a positive correlation in binding and neutralizing titers to most human strains, with the exception of the neutralizing titers to $\mathrm{H} 3 / 68$, which were 
Table 3. Multivariable Regression Analysis of Binding Titers With Respect to Age Group and Vaccination Status

\begin{tabular}{|c|c|c|c|}
\hline $\mathrm{rHA}$ & Characteristics & $\begin{array}{l}\text { Regression Coefficient } \\
(95 \% \mathrm{Cl})\end{array}$ & $\begin{array}{c}P \\
\text { Value }\end{array}$ \\
\hline \multirow[t]{3}{*}{$\mathrm{rH} 1 / 34$} & Vaccination $(\geq 1)$ & $0.35(.27-.43)$ & $<.001$ \\
\hline & Age (41-51) & $0.11(.016-.21)$ & .023 \\
\hline & Age (52-90) & $0.23(.13-.33)$ & $<.001$ \\
\hline \multirow[t]{3}{*}{$\mathrm{rH} 2 / 57$} & Vaccination $(\geq 1)$ & $0.12(-.10$ to .33$)$ & .267 \\
\hline & Age (41-51) & $0.53(.28-.78)$ & $<.001$ \\
\hline & Age (52-90) & $1.12(.86-1.38)$ & $<.001$ \\
\hline \multirow[t]{3}{*}{$\mathrm{rH} 3 / 99$} & Vaccination $(\geq 1)$ & $0.19(.070-.31)$ & .002 \\
\hline & Age (41-51) & $-0.11(-.26$ to .038$)$ & .145 \\
\hline & Age $(52-90)$ & $-0.25(-.40$ to -.095$)$ & .002 \\
\hline \multirow{3}{*}{$\begin{array}{l}\text { rH1 pdm/ } \\
09\end{array}$} & Vaccination $(\geq 1)$ & $0.20(.11-.29)$ & $<.001$ \\
\hline & Age (41-51) & $0.15(.048-.26)$ & .004 \\
\hline & Age (52-90) & $0.14(.033-.25)$ & .011 \\
\hline \multirow[t]{3}{*}{$\mathrm{rH} 4 / 56$} & Vaccination $(\geq 1)$ & $0.062(-.14$ to .26$)$ & .543 \\
\hline & Age (41-51) & $0.34(.096-.58)$ & .006 \\
\hline & Age (52-90) & $0.20(-.055$ to .45$)$ & .125 \\
\hline \multirow[t]{3}{*}{$\mathrm{rH} 5 / 04$} & Vaccination $(\geq 1)$ & $0.28(.16-.40)$ & $<.001$ \\
\hline & Age (41-51) & $0.35(.21-.49)$ & $<.001$ \\
\hline & Age (52-90) & $0.33(.18-.48)$ & $<.001$ \\
\hline \multirow[t]{3}{*}{$\mathrm{rH} 7 / 79$} & Vaccination $(\geq 1)$ & $-0.015(-.086$ to .055$)$ & .667 \\
\hline & Age (41-51) & $-0.041(-.13$ to .044$)$ & .347 \\
\hline & Age (52-90) & $-0.065(-.15$ to .020$)$ & .148 \\
\hline \multirow[t]{3}{*}{$\mathrm{rH} 12 / 76$} & Vaccination $(\geq 1)$ & $0.11(-.0037$ to .23$)$ & .058 \\
\hline & Age (41-51) & $-0.027(-.16$ to .11$)$ & .704 \\
\hline & Age (52-90) & $-0.11(-.25$ to .034$)$ & .052 \\
\hline
\end{tabular}

Reference age: 0-40 years.

Abbreviations: $\mathrm{Cl}$, confidence interval; rHA, recombinant hemagglutinin.

independent of vaccination (Figure 3 and Table 2). Titers to $\mathrm{H} 2 / 57$ were distinct, as binding titers did not depend on vaccination, and neutralizing titers showed a modest interaction between age and vaccination status. Specifically, neutralizing titers to $\mathrm{H} 2 / 57$ increased with age for both vaccinated and nonvaccinated groups, but were more pronounced in the vaccinated group. As both $\mathrm{H} 2$ and $\mathrm{H} 5$ subtypes have never been included in the vaccines, the fact that vaccination had a positive correlation with neutralizing titers to these subtypes can be explained by their phylogenetic proximity to the $\mathrm{H} 1$ subtype, which is part of the widely used split vaccine formulation.

To expose differences between age groups that would go undetected in linear regression, we performed Loess smoothing on antibody titers in relation to age separately for vaccinated and nonvaccinated donors. Neutralizing titers to recent human isolates ( $\mathrm{H} 1 / 07$ and $\mathrm{H} 3 / 07)$ were higher in vaccinees, but overall not influenced by age (as already suggested by linear regression). However, nonlinear regression revealed that the youngest participating donors (approximately $<30$ years) clearly showed the highest titers (Figure 3). Similar findings were made for
Table 4. Multivariable Regression Analysis of Neutralizing Titers With Respect to Age Group and Vaccination Status

\begin{tabular}{|c|c|c|c|}
\hline Virus & Characteristics & $\begin{array}{l}\text { Regression Coefficient } \\
\qquad(95 \% \mathrm{Cl})\end{array}$ & $\begin{array}{c}P \\
\text { Value }\end{array}$ \\
\hline \multirow[t]{3}{*}{$\mathrm{H} 1 / 34$} & Vaccination $(\geq 1)$ & $0.27(.15-.39)$ & $<.001$ \\
\hline & Age $(41-51)$ & $0.017(-.12$ to .16$)$ & .809 \\
\hline & Age (52-90) & $0.099(-.048$ to .25$)$ & .185 \\
\hline \multirow[t]{3}{*}{$\mathrm{H} 1 / 07$} & Vaccination $(\geq 1)$ & $0.66(.47-.85)$ & $<.001$ \\
\hline & Age $(41-51)$ & $-0.073(-.30$ to .15$)$ & .524 \\
\hline & Age (52-90) & $-0.074(-.31$ to .16$)$ & .536 \\
\hline \multirow[t]{3}{*}{$\mathrm{H} 2 / 57$} & Vaccination $(\geq 1)$ & $-0.085(-.18$ to .013$)$ & .089 \\
\hline & Age $(41-51)$ & $0.46(.34-.57)$ & $<.001$ \\
\hline & Age (52-90) & $0.96(.83-1.082)$ & $<.001$ \\
\hline \multirow[t]{3}{*}{$\mathrm{H} 3 / 68$} & Vaccination $(\geq 1)$ & $0.022(-.094$ to .14$)$ & .708 \\
\hline & Age (41-51) & $0.85(.71-.99)$ & $<.001$ \\
\hline & Age (52-90) & $0.73(.59-.88)$ & $<.001$ \\
\hline \multirow[t]{3}{*}{$\mathrm{H} 3 / 07$} & Vaccination $(\geq 1)$ & $0.40(.106-.69)$ & .008 \\
\hline & Age $(41-51)$ & $0.094(-.26$ to .45$)$ & .597 \\
\hline & Age (52-90) & $-0.096(-.46$ to .27$)$ & .604 \\
\hline \multirow[t]{3}{*}{$\mathrm{H} 4 / 56$} & Vaccination $(\geq 1)$ & $0.034(-.058$ to .13$)$ & .464 \\
\hline & Age (41-51) & $0.085(-.026$ to .20$)$ & .133 \\
\hline & Age (52-90) & $0.14(.021-.25)$ & .021 \\
\hline \multirow[t]{3}{*}{$\mathrm{H} 5 / 04$} & Vaccination $(\geq 1)$ & $3.43(.76-6.10)$ & .012 \\
\hline & Age $(41-51)$ & $0.71(-2.50$ to 3.92$)$ & .664 \\
\hline & Age (52-90) & $0.39(-2.94$ to 3.72$)$ & .818 \\
\hline \multirow[t]{3}{*}{$\mathrm{H} 7 / 79$} & Vaccination $(\geq 1)$ & $-1.059(-4.091$ to 1.97$)$ & .492 \\
\hline & Age (41-51) & $0.71(-2.95$ to 4.34$)$ & .708 \\
\hline & Age (52-90) & $3.32(-.64$ to 7.11$)$ & .085 \\
\hline
\end{tabular}

Reference age: 0-40 years.

Abbreviation: $\mathrm{Cl}$, confidence interval.

binding titers to recently isolated $\mathrm{H} 3 / 99$. This was in contrast to the behavior observed against older isolates (H2/57 and $\mathrm{H} 3 / 68$ ); here the best neutralizing titers were found in those donors who were in their first decade of life when the corresponding isolate had been circulating (ages 50-61 and 41-50, respectively). The same trend emerged for binding titers to $\mathrm{H} 2 / 57$. In contrast, for an older $\mathrm{H} 1$ isolate $(\mathrm{H} 1 / 34)$, this effect was less pronounced for both neutralizing and binding titers.

\section{DISCUSSION}

Our study demonstrates that heterosubtypic antibodies against influenza viruses-that is, antibodies recognizing hemagglutinin from multiple subtypes-can be found in most exposed or immunized individuals. These findings corroborate previous findings made by biochemical analysis of a limited number of samples $[4,8]$. We have found that vaccination not only enhanced antibody responses to subtypes that are currently circulating, and are therefore included in the vaccine, but also augmented binding and neutralizing antibody titers to 
A

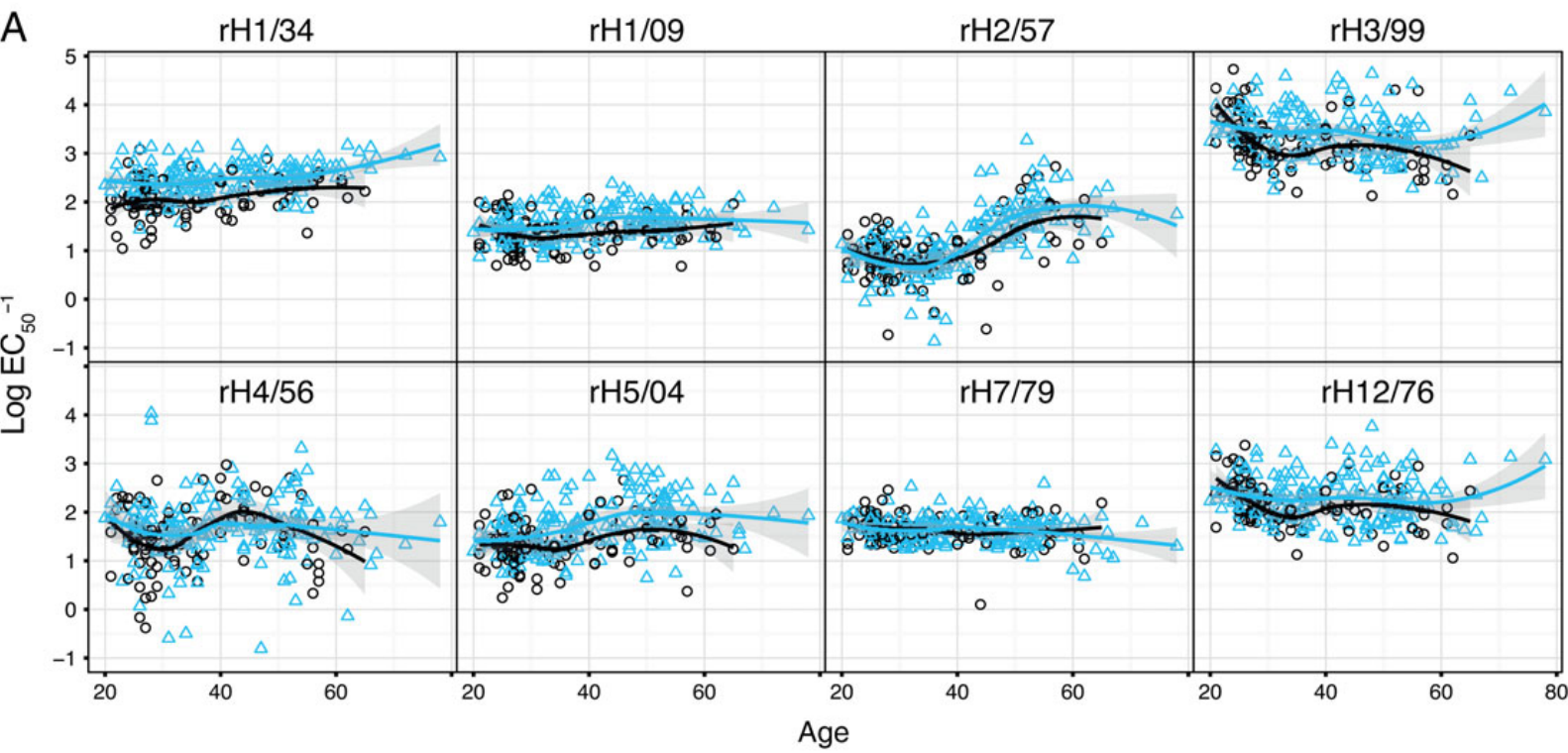

B

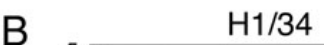

$\mathrm{H} 1 / 07$

$\mathrm{H} 2 / 57$

$\mathrm{H} 3 / 68$

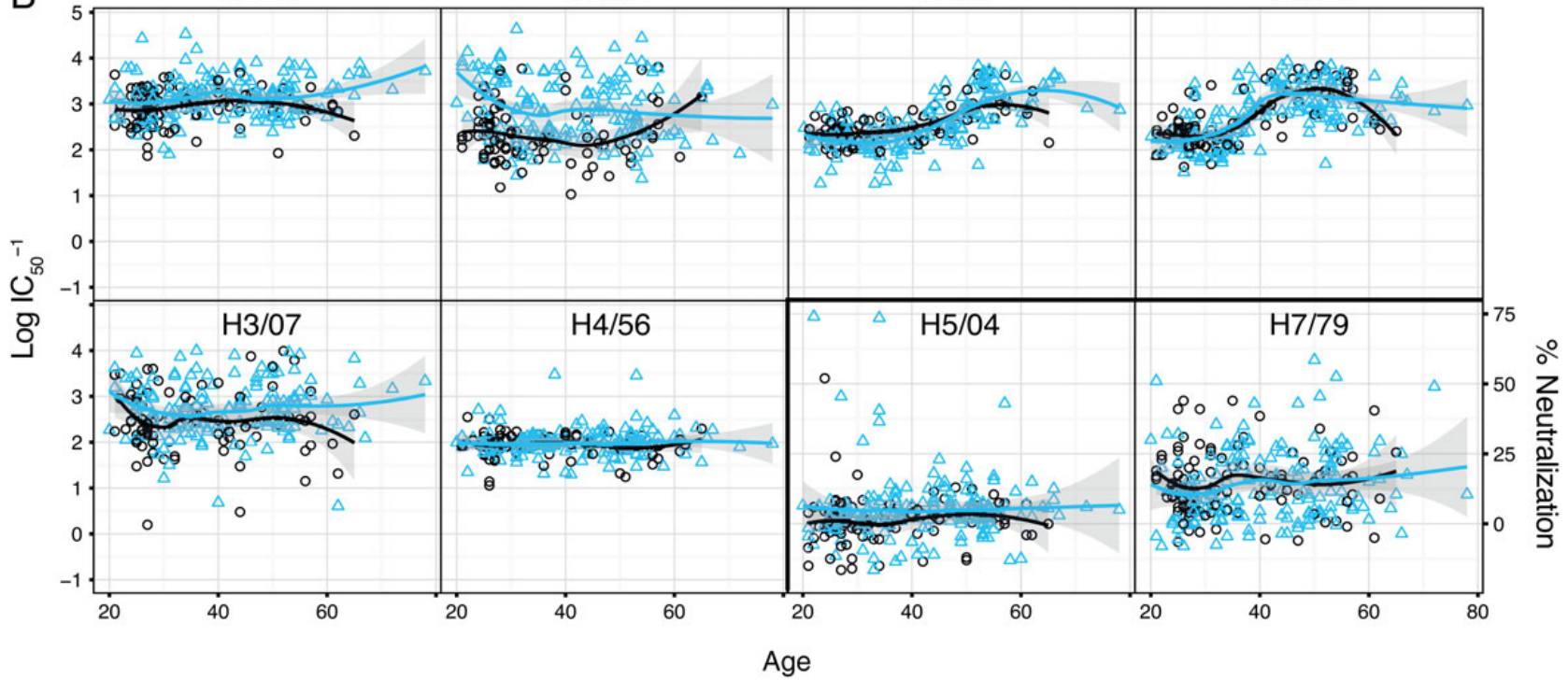

Figure 3. Loess smoothing of antibody titers. $A$, The binding antibody titers for $\mathrm{rH} 3 / 99$, but not for $\mathrm{rH} 1 / 34$ or $\mathrm{rH} 2 / 57$, were lower in more seasoned individuals. Smoothing discovers steep increase of $-\log \left(\mathrm{EC}_{50}\right)$ around age 40 for $\mathrm{rH} / 57$. For $\mathrm{rH} 1 / 34$, smoothing displayed results similar to linear regression. Highest $-\log \left(\mathrm{EC}_{50}\right)$ titers to $\mathrm{rH3} / 99$ are depicted in individuals aged 21-27 years. Values are shown as logarithm of the reciprocal serum dilution in correlation with age. $B$, Neutralizing titers to $\mathrm{H} 1 / 34$ (vaccinated), $\mathrm{H} 2 / 57$ (vaccinated and nonvaccinated), and $\mathrm{H} 3 / 68$ (vaccinated and nonvaccinated) increased with increasing age. The - $\log \left(\mathrm{IC}_{50}\right)$ to $\mathrm{H} 1 / 07$ (vaccinated) decreased with increasing age (compare with Supplementary Figure 2). Remaining subtypes do not show slopes different from zero. Smoothing of $-\log \left(\mathrm{IC}_{50}\right)$ for $\mathrm{H} 2 / 57$ discovered a peak at age 50 . Values are shown as logarithm of the reciprocal serum dilution giving a half-maximal inhibitory concentration in correlation with age, and, separated by bold frame lines, as inhibition percentage against $\mathrm{H} 5 / 57$ and $\mathrm{H7/79}$ at a serum dilution of 1 in 90 . Blue triangles and lines represent the vaccinated cohort; black squares and lines represent the nonvaccinated cohort. Gray shaded areas indicate the confidence band. Significance codes (testing the slope being different from zero): ${ }^{* * *} P<.001$, ${ }^{*} P<.01,{ }^{*} P<.05$. Abbreviations: $\mathrm{EC}_{50}$, half-maximal effective concentration; $\mathrm{I}_{50}$, half-maximal inhibitory concentration.

heterosubtypic subtypes. Senior people had higher antibody titers to old homosubtypic influenza isolates than their younger counterparts, whose antibody response appeared to favor more recent isolates. Age was also found to be a predictor with a positive coefficient for higher antibody titers to heterosubtypic isolates. In contrast, the number of self-reported influenza 
episodes did not correlate with higher antibody titers, which, given the unreliability of the parameter, is not surprising. Moreover, because vaccinations, like influenza episodes, also accumulate with age and may not always be reported accurately, it is hard to disentangle their effect on the antibody titers. For this reason, vaccination status was reported as binary (vaccinated/nonvaccinated), discarding differences in the number of vaccinations.

As the participants of our study have not recently been vaccinated, their antibody repertoire is not skewed toward a recently inoculated vaccine strain. Attention is therefore focused on the serum antibody composition present when infection is most likely to occur. The use of viable viruses for neutralization assays rather than highly neutralization-sensitive pseudotyped virions further assured that the neutralizing activity detected is biologically relevant [9].

At the time of serum sampling, H1pdm/09 had not yet reached Switzerland. Potential H1pdm/09-related induction of broadly cross-reactive antibodies [10] is therefore unlikely to have skewed our results. Yet, our data are consistent with findings that $\mathrm{H} 1 \mathrm{pdm} /$ 09 possesses an epitope that is shared with the H1N1 virus strains circulating before 1957; individuals born before 1957 had higher titers to H1pdm/09 than younger participants [11, 12]. Although the binding titers were expected to increase with age in the nonvaccinated cohort, no peak in elderly persons appeared in nonparametric smoothing. Both observations, however, could be explained by the low sample size in this age group (Figure 3 ).

Because we used a novel neutralization assay, we cannot give predictions for protection. In the hemagglutinin inhibition assays (HIAs), a serum dilution of 1:40 is considered protective whereas comparable values were found for an ELISA-based and a colorimetric microneutralization assay; however, these values are not applicable to our assay [13], in particular as it was also shown that ELISA values and HIA values do not correlate, not even within the same subtypes [14]. Moreover, because the majority of heterosubtypic antibodies was found to bind a conserved epitope in the stem of the HA protein and did not interfere with hemagglutination $[15,16]$, they would not be detected by HIAs. Consequently, no direct predictions for heterosubtypic protection can be drawn from our dataset at this point. Further research will be required to define this relationship.

In contrast, the large number of serum samples tested for both binding and neutralization allowed us the establishment of a linear relationship: Increasing titers of binding antibodies correlate statistically significant with increasing neutralizing titers (Supplementary Table 2). The correlation coefficient for extinct human strains was reasonably high, especially in $\mathrm{rH} 2 / 57$ vs $\mathrm{H} 2 / 57(R=0.43)$, whereas isolates with very distant isolation years (H3/68 vs $\mathrm{rH} 3 / 99)$ did not correlate at all, despite belonging to the same subtype.

Lessler and colleagues found that repeated exposure to different $\mathrm{H} 3 \mathrm{~N} 2$ strains increased antibody titers to those strains encountered earlier in life, whereas progressively fewer specific antibodies to subsequent infection are made with age [3]. We could confirm this finding in that the neutralizing and binding titers were the highest in those individuals who were in their first decade of life during the period of time when the corresponding subtype was circulating. Older individuals also had significantly more binding and neutralizing antibodies against old than against more recent isolates (eg, H3/99; Supplementary Figure 2), which may reflect original antigenic sin [17]. In contrast, in line with other studies [18], we found antibody titers to the last prestudy isolates (2007) to be the same in all age groups.

In elderly individuals, vaccination increased the probability of having heterosubtypic antibodies. Thus, whereas senescence of the immune system presumably contributed to a poorer response to recent isolates, age was advantageous for the development of heterosubtypic antibodies (to both human and nonhuman isolates). Vaccination always improved titers in the oldest or middle age group compared with young individuals. Although the age at which the vaccinations were received was not assessed, most individuals received them in the previous $5-10$ years. In fact, influenza vaccination was very uncommon in Switzerland prior to the late 1990s [19]. Our findings show that vaccination is of value also in more seasoned individuals, and that its benefit is not just the result of vaccinations at younger age. This provides additional, albeit indirect, support for annual vaccinations in elderly individuals.

Although most humans have low levels of heterosubtypic antibodies, they are still susceptible to infection with antigenically drifted or shifted influenza A strains, indicating that these antibodies probably are not protective. However, such antibodies are likely to attenuate disease, but to prove this hypothesis, large clinical trials would need to be conducted. The findings of our study nonetheless clearly indicate that novel vaccination strategies targeting the conserved epitopes of influenza hemagglutinin could profit from preexisting antigen-experienced heterosubtypic B cells. Our data also unambiguously support yearly vaccination as neutralizing antibody titers increase over time in elderly and younger people and, furthermore, as more heterosubtypic antibodies are induced.

\section{Supplementary Data}

Supplementary materials are available at Clinical Infectious Diseases online (http://cid.oxfordjournals.org). Supplementary materials consist of data provided by the author that are published to benefit the reader. The posted materials are not copyedited. The contents of all supplementary data are the sole responsibility of the authors. Questions or messages regarding errors should be addressed to the author.

\section{Notes}

Acknowledgments. We are grateful to all of the study participants; the donors; the physicians; and the study nurses, especially Christina Grube, Division of Infectious Diseases and Hospital Epidemiology, University Hospital Zurich. We thank Alexandra Trkola, Jacqueline Weber, Peter Rusert, 
Irene Abela, Doris Jeannette, and Therese Uhr from the Institute of Medical Virology for their help, and the Life Science Zurich Graduate School for their support. We also express our gratitude to Drs Richard Webby and Scott Kraus from the St Jude Children's Research Hospital, Memphis, Tennessee; Yves Thomas and Laurent Kaiser from the University Hospital of Geneva, Switzerland; and Rodney Daniels from the National Institute of Medical Research, London, UK, for kindly providing viruses from their repository.

Financial support. This work was supported by the Swiss National Science Foundation (grant numbers PP00P3_123429, PP00P3_146345 to L. H. and 130865 to H. F. G.). These grants also provided funding for I. K., M. B., A. W., and M. S. (employed by L. H.), and A. U. S. (employed by $\mathrm{H}$. G.).

Potential conflicts of interest. O. Z. is employed by the University of Zurich, and B. L. is employed by the University Hospital of Zurich. All other authors report no potential conflicts.

All authors have submitted the ICMJE Form for Disclosure of Potential Conflicts of Interest. Conflicts that the editors consider relevant to the content of the manuscript have been disclosed.

\section{References}

1. Smith DJ, Lapedes AS, de Jong JC, et al. Mapping the antigenic and genetic evolution of influenza virus. Science 2004; 305:371-6.

2. Angelova LA, Shvartsman YaS. Original antigenic sin to influenza in rats. Immunology 1982; 46:183-8.

3. Lessler J, Riley S, Read JM, et al. Evidence for antigenic seniority in influenza A (H3N2) antibody responses in southern China. PLoS Pathog 2012; 8:e1002802.

4. Sui J, Sheehan J, Hwang WC, et al. Wide prevalence of heterosubtypic broadly neutralizing human anti-influenza A antibodies. Clin Infect Dis 2011; 52:1003-9.

5. Davenport FM, Hennessy AV, Francis T Jr. Epidemiologic and immunologic significance of age distribution of antibody to antigenic variants of influenza virus. J Exp Med 1953; 98:641-56.

6. Francis T Jr. On the doctrine of original antigenic sin. Proc Am Philos Soc 1960; 104:572-8.

7. Ochsenbein AF, Zinkernagel RM. Natural antibodies and complement link innate and acquired immunity. Immunol Today 2000; 21: 624-30.
8. Corti D, Suguitan AL Jr, Pinna D, et al. Heterosubtypic neutralizing antibodies are produced by individuals immunized with a seasonal influenza vaccine. J Clin Invest 2010; 120:1663-73.

9. Garcia J-M, Lai JCC. Production of influenza pseudotyped lentiviral particles and their use in influenza research and diagnosis: an update. Expert Rev Anti Infect Ther 2011; 9:443-55.

10. Li GM, Chiu C, Wrammert J, et al. Pandemic H1N1 influenza vaccine induces a recall response in humans that favors broadly cross-reactive memory B cells. Proc Natl Acad Sci U S A 2012; 109: 9047-52.

11. Hancock K, Veguilla V, Lu X, et al. Cross-reactive antibody responses to the 2009 pandemic H1N1 influenza virus. N Engl J Med 2009; 361:1945-52.

12. Xu R, Ekiert DC, Krause JC, Hai R, Crowe JE, Wilson IA. Structural basis of preexisting immunity to the $2009 \mathrm{H} 1 \mathrm{~N} 1$ pandemic influenza virus. Science 2010; 328:357-60.

13. Grund S, Adams O, Wahlisch S, Schweiger B. Comparison of hemagglutination inhibition assay, an ELISA-based micro-neutralization assay and colorimetric microneutralization assay to detect antibody responses to vaccination against influenza A H1N1 2009 virus. J Virol Methods 2011; 171:369-73.

14. Ducatez MF, Bahl J, Griffin Y, et al. Feasibility of reconstructed ancestral $\mathrm{H} 5 \mathrm{~N} 1$ influenza viruses for cross-clade protective vaccine development. Proc Natl Acad Sci U S A 2011; 108:349-54.

15. Wyrzucki A, Dreyfus C, Kohler I, Steck M, Wilson IA, Hangartner L. Alternative recognition of the conserved stem epitope in influenza $\mathrm{A}$ virus hemagglutinin by a $\mathrm{VH} 3-30$-encoded heterosubtypic antibody. J Virol 2014; 88:7083-92.

16. Dreyfus C, Laursen NS, Kwaks T, et al. Highly conserved protective epitopes on influenza B viruses. Science 2012; 337:1343-8.

17. Lambert PH, Liu M, Siegrist CA. Can successful vaccines teach us how to induce efficient protective immune responses? Nat Med 2005; 11 (4 suppl):S54-62.

18. Powers DC, Belshe RB. Vaccine-induced antibodies to heterologous influenza A H1N1 viruses: effects of aging and 'original antigenic sin.' J Infect Dis 1994; 169:1125-9.

19. Voordouw AC, Sturkenboom MC, Dieleman JP, et al. Annual revaccination against influenza and mortality risk in community-dwelling elderly persons. JAMA 2004; 292:2089-95. 